\title{
Pseudo-Exponential-Type Solutions of Wave Equations Depending on Several Variables
}

\author{
Bernd FRITZSCHE ${ }^{\dagger}$, Bernd KIRSTEIN ${ }^{\dagger}$, Inna Ya. ROITBERG ${ }^{\dagger}$ \\ and Alexander L. SAKHNOVICH ${ }^{\ddagger}$ \\ $\dagger$ Fakultät für Mathematik und Informatik, Universität Leipzig, \\ Augustusplatz 10, D-04009 Leipzig, Germany \\ E-mail: fritzsche@math.uni-leipzig.de,kirstein@math.uni-leipzig.de,innaroitberg@gmail.com \\ $\ddagger$ Fakultät für Mathematik, Universität Wien, \\ Oskar-Morgenstern-Platz 1, A-1090 Vienna, Austria \\ E-mail: oleksandr.sakhnovych@univie.ac.at
}

Received September 04, 2014, in final form January 23, 2015; Published online January 29, 2015

http://dx.doi.org/10.3842/SIGMA.2015.010

\begin{abstract}
Using matrix identities, we construct explicit pseudo-exponential-type solutions of linear Dirac, Loewner and Schrödinger equations depending on two variables and of nonlinear wave equations depending on three variables.

Key words: Bäcklund-Darboux transformation; matrix identity; $S$-node; $S$-multinode; explicit solution; non-stationary Dirac equation; non-stationary Schrödinger equation; Loewner system; pseudo-exponential-type potential; integrable nonlinear equations
\end{abstract}

2010 Mathematics Subject Classification: 35C08; 35Q41; 15A24

\section{Introduction}

The term pseudo-exponential potentials was introduced in [20] (see Remark 1.2 on interrelations between pseudo-exponential-type potentials and multi-soliton solutions). Ordinary linear differential equations with the so called pseudo-exponential-type potentials were actively studied (see [14, 15, 20, 21, 22, 38, 41] and references therein), since their solutions could be constructed explicitly (and inverse problems to recover these equations from rational Weyl functions or reflection coefficients could be solved explicitly). Thus, pseudo-exponential-type potentials and solutions, that is, potentials and solutions, which, roughly speaking, rationally depend on matrix exponentials, are of a special interest. When matrices in the matrix exponentials (from the rational functions of matrix exponentials) are nilpotent, purely rational functions (potentials) appear as an important subcase of the pseudo-exponential-type potentials. For a more rigorous definition of the term pseudo-exponential potential see, for example, [15, 20].

Explicit solutions of linear and nonlinear wave equations are important both in theory and applications. The theory is well-developed for the case of linear equations depending on one variable and nonlinear integrable equations depending on two variables and includes, in particular, algebro-geometric methods and several versions of the commutation methods and of Bäcklund-Darboux transformations (BDTs), see some results and various references in $[10,12,16,17,18,23,32,41,52]$. In spite of numerous interesting results on the cases of more variables (see, e.g., $[1,5,7,8,13,31,33,34,37,46,48,49]$ ), these cases are more complicated and contain also more open problems.

Matrix identities are actively used in this theory for the cases of one and several space variables starting from the seminal work [30]. By matrix (or operator) identities we mean an important subclass of so called Sylvester equations $A X-Y B=Q$, which are considered, for 
instance, in control theory. Namely, matrix identities are equations of the form $A R-R B=Q$ or, more often, $A R-R B=\Pi_{1} \Pi_{2}^{*}$ (see, e.g., $[35,42,44]$ ) with $\Pi_{k}$ of comparatively small rank. V.A. Marchenko [30] was the first to apply matrix and operator identities in this topic (see [46] and references therein for further developments of his approach). In another way (more precisely, for the construction of $\tau$-functions) matrix identities were used in [25]. Our approach is based on the GBDT (generalized BDT) approach, which was introduced in [35, 36] (see further results and many references in [14, 15, 20, 39, 41]). Although the papers [35, 36] were initiated by [30], matrix identities in [30] and in GBDT are used in quite different ways. Moreover, solutions of the nonlinear equations are constructed in [30] as reductions of expressions of the form $\Gamma^{-1} \Gamma_{x}$ whereas GBDT is a kind of a binary Darboux transformation and solutions are expressed via matrix functions $\Phi_{2}^{*} S^{-1} \Phi_{1}$. (Here $\Gamma, \Phi_{1}$ and $\Phi_{2}$ satisfy some simple auxiliary linear systems.) See, for instance, (4.5) for solutions in terms of $\Phi_{2}^{*} S^{-1} \Phi_{1}$. Matrices of a much lesser order have to be inverted in GBDT when constructing, for instance, matrix solutions of nonlinear equations. In addition, Darboux matrices and wave functions are constructed explicitly using GBDT. The method develops during the last 20 years. Moreover, after the publication of [35, 36] a very close approach was used by M. Manas (see some comparative analysis in [10]) and related formulas are now successfully used by Mueller-Hoissen and coauthors (see, e.g., [13]).

In our paper we apply multidimensional versions of the GBDT. That is, we follow [37] (where $S$-nodes introduced in $[42,43,44]$ were applied to matrix Kadomtsev-Petviashvili equations) and the $S$-multinodes approach from [40] in order to construct explicitly pseudo-exponential-type potentials and solutions of some important equations of mathematical physics depending on several variables. The transfer to $S$-multinodes is required in many examples because the same matrix should satisfy several matrix identities. $S$-multinodes first appeared in [40] as a certain generalization of the $S$-nodes on one hand and commutative colligations (introduced by M.S. Livšic [27]) on the other hand.

A symmetric $S$-multinode ( $r$-node) is a set of matrices

$$
\left\{A_{1}, \ldots, A_{r} ; \nu_{1}, \ldots, \nu_{r} ; R ; \widehat{C}\right\}
$$

such that for $1 \leq i, k \leq r$ the relations

$$
A_{i} A_{k}=A_{k} A_{i}, \quad A_{k} R+R A_{k}^{*}=\widehat{C} \nu_{k} \widehat{C}^{*}, \quad R=R^{*}, \quad \nu_{k}=\nu_{k}^{*}
$$

hold. Here we shall deal with the cases $r=1,2,3$. In the case $r=1$ we have the well-known symmetric $S$-node introduced by L.A. Sakhnovich (see, e.g., [41, 42, 43, 44, 45] for various applications). For $r>1$ the situation is more complicated, since $R$ in general position is defined already by one of the identities $A_{k} R+R A_{k}^{*}=\widehat{C} \nu_{k} \widehat{C}^{*}$. However, the construction of $S$-multinodes proves both possible and useful.

Remark 1.1. In our further considerations the matrices in the $S$-multinode or $S$-node (i.e., matrices in (1.1)) are constant and each $S$-multinode generates a potential and solution of a linear (or solution of a nonlinear) equation.

Remark 1.2. We note that pseudo-exponential-type solutions are close to multi-soliton solutions and their analogues. However, multi-soliton solutions are usually generated when matrices $A_{i}$ are diagonal, whereas we do not require $A_{i}$ to be necessarily diagonal. This correspondence for the solutions of sine-Gordon and sinh-Gordon equations was studied in [35, Section 4]. In particular, it was shown in [35] that solutions of sine-Gordon equation from [9, 24] are derived in this way (i.e., using $S$-nodes with diagonal matrices $A_{1}$ ).

Explicit solutions of linear equations (especially, of non-stationary Dirac and Schrödinger equations) are of wide interest, and in Section 2 we use 2-nodes in order to study the case of 
the non-stationary Dirac system

$$
H \Psi=0, \quad H:=\frac{\partial}{\partial t}+\sigma_{2} \frac{\partial}{\partial y}-\mathrm{i} V(t, y), \quad \sigma_{2}=\left[\begin{array}{cc}
0 & -\mathrm{i} \\
\mathrm{i} & 0
\end{array}\right], \quad V=V^{*},
$$

which presents more difficulties than the non-stationary (time-dependent) Schrödinger equation considered in [40]. Some new results for the non-stationary Schrödinger equation are derived in Section 3. Thus, we fill in the gap between papers [37] and [40], consider a class of solutions of the Schrödinger equation, which is wider than the one discussed in [1], and construct interesting examples.

Section 4 is dedicated to the nonlinear integrable equations. As examples we consider matrix Davey-Stewartson I (DS I) and generalized nonlinear optics equations. In particular, our approach allows to construct a wide class of rational solutions of matrix DS I (see Remark 4.3).

Remark 1.3. GBDT results for DS I and generalized nonlinear optics equation were obtained in [36, Section 3] but no examples were given. Here we construct wide classes of solutions using the $S$-node ( $S$-multinode) approach, see Propositions 4.2 and 4.6. We note that GBDT results in [36, Section 3] include the case of nonzero background (in which situation auxiliary linear systems play a more essential role) and it would be very interesting to generalize $S$-multinode approach for that case.

As usual, $\mathbb{N}$ denotes the set of natural numbers, const stands for a constant (number or matrix), $\operatorname{Im}(A)$ stands for the image of the matrix $A, \sigma(D)$ stands for the spectrum of $D,[G, F]$ stands for the commutator $G F-F G, \otimes$ stands for Kronecker product, $I_{p}$ is the $p \times p$ identity matrix, and $\Psi_{t x}:=\frac{\partial}{\partial x}\left(\frac{\partial}{\partial t} \Psi\right)=\frac{\partial^{2}}{\partial x \partial t} \Psi$. By $\operatorname{diag}\left\{b_{1}, b_{2}, \ldots, b_{m}\right\}$ we denote the diagonal matrix with the entries $b_{1}, b_{2}, \ldots$ on the main diagonal.

\section{Dirac and Loewner equations: explicit solutions}

\subsection{Non-stationary Dirac equation}

We note that in the GBDT version of the Bäcklund-Darboux transformation the solution of the transformed equation is represented in the form $\Pi^{*} S^{-1}$, where $\Pi^{*}$ is a matrix solution of the initial equation and the matrix function $S$ is constructed using the $S$-node (see, e.g., [39, 41] and references therein). Here we construct solutions of (1.2) in the same form. Namely, we set

$$
\Pi=C E_{A}(t, y) \widehat{C}, \quad E_{A}=\exp \left\{t A_{1}+y A_{2}\right\}, \quad A_{1} A_{2}=A_{2} A_{1}, \quad \widehat{C}=\left[\begin{array}{ll}
g_{1}^{*} & g_{2}^{*}
\end{array}\right],
$$

where $\widehat{C}$ is an $N \times 2$ matrix, $g_{1}^{*}$ and $g_{2}^{*}$ are columns of $\widehat{C}, A_{1}$ and $A_{2}$ are $N \times N$ matrices and $C$ is an $n \times N$ matrix $(n, N \in \mathbb{N})$. We emphasize that the matrices $A_{1}, A_{2}, \widehat{C}$ and $C$ are constant (see also Remark 1.1). We assume that the equalities

$$
g_{1} A_{1}^{*}-\mathrm{i} g_{2} A_{2}^{*}=0, \quad g_{2} A_{1}^{*}+\mathrm{i} g_{1} A_{2}^{*}=0
$$

hold. From (2.1) and (2.2), we easily see that

$$
H_{0} \Pi^{*}=0, \quad H_{0}:=\frac{\partial}{\partial t}+\sigma_{2} \frac{\partial}{\partial y},
$$

where $H_{0}$ is applied to $\Pi^{*}$ columnwise.

Recall that matrices $A_{1}, A_{2}, R, \nu_{1}, \nu_{2}$ and $\widehat{C}$ form a symmetric 2-node if $A_{1}$ and $A_{2}$ commute and the following identities are valid:

$$
A_{k} R+R A_{k}^{*}=\widehat{C} \nu_{k} \widehat{C}^{*}, \quad k=1,2, \quad R=R^{*}, \quad \nu_{k}=\nu_{k}^{*} .
$$


It is immediate that the matrix function

$$
S(t, y)=S_{0}+C E_{A}(t, y) R E_{A}(t, y)^{*} C^{*}, \quad S_{0}=S_{0}^{*} \equiv \text { const },
$$

satisfies equations $\frac{\partial}{\partial t} S=\Pi \nu_{1} \Pi^{*}$ and $\frac{\partial}{\partial y} S=\Pi \nu_{2} \Pi^{*}$. These equations and equation (2.3) yield the proposition below.

Proposition 2.1. Let relations (2.1), (2.2), (2.4) and (2.5) hold and assume that $\nu_{1}=\sigma_{2}$, $\nu_{2}=-I_{2}$. Then, in the points of invertibility of $S$, we have

$$
H\left(\Pi(t, y)^{*} S(t, y)^{-1}\right)=0,
$$

where $H$ has the form (1.2) with $V$ defined by

$$
V:=\mathrm{i}\left(\Pi^{*} S^{-1} \Pi \sigma_{2}-\sigma_{2} \Pi^{*} S^{-1} \Pi\right) .
$$

The important part of the problem is to find the cases where the conditions of Proposition 2.1 hold. Then we obtain families of explicitly constructed potentials $V$ and solutions $\Pi^{*} S^{-1}$ of the corresponding Dirac systems.

Example 2.2. Set $g_{2}=-\mathrm{i} g_{1} j_{n}, A_{1}=D=\operatorname{diag}\left\{D_{1}, D_{2}\right\}$ (where $D_{1}$ and $D_{2}$ are $n_{1} \times n_{1}$ and $n_{2} \times n_{2}$ diagonal blocks of the diagonal matrix $D, n_{1}+n_{2}=n, \sigma\left(D_{k}\right) \cap \sigma\left(-D_{k}^{*}\right)=\varnothing$ for $\left.k=1,2\right)$, $A_{2}=D j_{n}$ and

$$
j_{n}:=\left[\begin{array}{cc}
I_{n_{1}} & 0 \\
0 & -I_{n_{2}}
\end{array}\right], \quad R=\left[\begin{array}{cc}
R_{11} & 0 \\
0 & R_{22}
\end{array}\right] .
$$

We uniquely define $R_{11}$ and $R_{22}$ by the matrix identities

$$
D_{1} R_{11}+R_{11} D_{1}^{*}=-g_{1}^{*}\left(I_{n}+j_{n}\right) g_{1}, \quad D_{2} R_{22}+R_{22} D_{2}^{*}=g_{1}^{*}\left(I_{n}-j_{n}\right) g_{1} .
$$

Then the conditions of Proposition 2.1 hold.

Thus, according to Proposition 2.1 and Example 2.2, each vector $g_{1}$ and diagonal matrix $D$ (such that $\sigma\left(D_{k}\right) \cap \sigma\left(-D_{k}^{*}\right)=\varnothing$ ) determine a set (depending on the choice of $C$ and $S_{0}$ ) of pseudo-exponential-type potentials and explicit solutions of (1.2).

\subsection{Loewner's system}

Loewner's system has the form

$$
\Psi_{x}=\mathcal{L}(x, y) \Psi_{y}
$$

where $\mathcal{L}$ is an $m \times m$ matrix function. For the case $m=2$, this system was studied by C. Loewner in the seminal paper [28] and applications to the hodograph equation were obtained. In [29], C. Loewner rewrote in this way the system $x_{\eta}-y_{\xi}=0,(\rho x)_{\xi}+(\rho y)_{\eta}=0$, which describes a steady compressible and irrotational flow of an ideal fluid. For the Loewner's system, its transformations, generalizations and applications, see also $[47,50]$ and references therein. (For some special kinds of similarity transformations of $\mathcal{L}$ see also [28, formulas (5.10a) and (5.27)].) Direct calculation proves the following proposition.

Proposition 2.3. Let $m \times m$ and $m \times n$, respectively, matrix functions $\Lambda_{1}$ and $\Lambda_{2}$ satisfy a linear differential equation

$$
\left(\Lambda_{i}\right)_{x}=q_{1}(x, y)\left(\Lambda_{i}\right)_{y}+q_{0}(x, y) \Lambda_{i}, \quad i=1,2,
$$

where the coefficients $q_{0}$ and $q_{1}$ are some $m \times m$ matrix functions. Then, in the points of invertibility of $\Lambda_{1}$, the matrix function $\Psi=\Lambda_{1}^{-1} \Lambda_{2}$ satisfies the Loewner equation (2.6), where

$$
\mathcal{L}=\Lambda_{1}^{-1} q_{1} \Lambda_{1} .
$$


Pseudo-exponential-type $\Psi$ and $\mathcal{L}$ are constructed in the next proposition.

Proposition 2.4. Introduce $m \times m$ and $m \times n$, respectively, matrix functions $\Lambda_{1}$ and $\Lambda_{2}$ by the equalities

$$
\begin{aligned}
& \Lambda_{i}=\mathcal{C}_{i} E_{A}(x, y, i) \widehat{\mathcal{C}}_{i}, \quad i=1,2, \\
& E_{A}(x, y, i):=\exp \left\{x \breve{A}_{i}+y \widetilde{A}_{i}\right\}, \quad \breve{A}_{i}:=D \otimes A_{i}, \quad \widetilde{A}_{i}:=I_{m} \otimes A_{i}, \\
& D=\operatorname{diag}\left\{d_{1}, \ldots, d_{m}\right\}, \quad \mathcal{C}_{i}:=\sum_{k=1}^{m}\left(e_{k} e_{k}^{*}\right) \otimes\left(e_{k}^{*} c_{i}\right),
\end{aligned}
$$

where $A_{i}$ are $l_{i} \times l_{i}$ matrices, $c_{i}$ are $m \times l_{i}$ matrices, $\widehat{\mathcal{C}_{1}}$ is an $N_{1} \times m$ matrix, $\widehat{\mathcal{C}_{2}}$ is an $N_{2} \times n$ matrix, $N_{i}=m l_{i}$ and $l_{i} \in \mathbb{N}$. Here $\otimes$ is Kronecker product, $e_{k}$ is a column vector given by $e_{k}=\left\{\delta_{j k}\right\}_{j=1}^{m}$ and $\delta_{j k}$ is Kronecker's delta.

Then, in the points of invertibility of $\Lambda_{1}$, the matrix functions

$$
\Psi=\Lambda_{1}^{-1} \Lambda_{2} \quad \text { and } \quad \mathcal{L}=\Lambda_{1}^{-1} D \Lambda_{1}
$$

satisfy (2.6).

Proof. It is easy to see that $\Lambda_{1}$ and $\Lambda_{2}$ given by (2.7) satisfy equation $\left(\Lambda_{i}\right)_{x}=D\left(\Lambda_{i}\right)_{y}$. Now, Proposition 2.4 follows from Proposition 2.3.

In a similar (to the construction of $\Lambda_{i}$ in the proposition above) way, matrix functions $\Pi$ satisfying (4.19) are constructed in (4.21)-(4.23).

\section{Non-stationary Schrödinger equation: explicit solutions and examples}

We consider the subcase of [40, Theorem 3.2], where $\mathcal{S}_{0}=\mathcal{S}_{0}^{*}$, and use notations $\Pi$ instead of $\Psi_{0}$, $S$ instead of $\mathcal{S}$ and $S_{0}$ instead of $\mathcal{S}_{0}$. We substitute

$$
\begin{aligned}
& \alpha=\mathrm{i}, \quad k=1, \quad A_{1}=A, \quad B_{1}=-A^{*}, \quad \nu_{1}=I_{p}, \\
& C_{\Phi}=\widehat{C}, \quad C_{\Psi}=\widehat{C}^{*}, \quad \widehat{C}_{\Phi}=C, \quad \widehat{C}_{\Psi}=C^{*}
\end{aligned}
$$

into [40, formula (3.1) and Theorem 3.2]. For this particular case, Theorem 3.2 from [40] takes the following form.

Proposition 3.1. Fix some $p, n, N \in \mathbb{N}$, an $N \times N$ matrix $A$, an $n \times N$ matrix $C$, an $N \times p$ matrix $\widehat{C}$ and an $n \times n$ matrix $S_{0}=S_{0}^{*}$. Let $R=R^{*}$ satisfy the matrix identity

$$
A R+R A^{*}=\widehat{C} \widehat{C}^{*},
$$

and put

$$
\begin{aligned}
& \Pi(x, t)=C e_{A}(x, t) \widehat{C}, \quad e_{A}(x, t):=\exp \left\{x A-\mathrm{i} t A^{2}\right\}, \\
& S(x, t)=S_{0}+C e_{A}(x, t) R e_{A}(x, t)^{*} C^{*} .
\end{aligned}
$$

Then, the matrix function $\widetilde{\Pi}^{*}:=\Pi^{*} S^{-1}$ satisfies the vector non-stationary Schrödinger equation

$$
H\left(\widetilde{\Pi}^{*}\right)=0, \quad H:=\mathrm{i} \frac{\partial}{\partial t}+\frac{\partial^{2}}{\partial x^{2}}-\widetilde{q}(x, t),
$$

where $H$ is applied to $\widetilde{\Pi}^{*}$ columnwise and $\widetilde{q}$ is the $p \times p$ matrix function:

$$
\widetilde{q}(x, t)=-2\left(\Pi(x, t)^{*} S(x, t)^{-1} \Pi(x, t)\right)_{x} .
$$


Our approach allows to consider the cases of non-diagonal matrices $A$, and we adduce below several examples, where $A$ is a $2 \times 2$ Jordan cell. Using some simple calculations, we easily construct $e_{A}, \Pi, S$ and, finally, solution $\widetilde{\Pi}^{*}$ and potential $\widetilde{q}$ in the following example of a scalar Schrödinger equation.

Example 3.2. Let us put

$$
p=1, \quad N=n=2, \quad A=\left[\begin{array}{cc}
\mu_{0} & 1 \\
0 & \mu_{0}
\end{array}\right], \quad \widehat{C}=\left[\begin{array}{l}
\widehat{c}_{1} \\
\widehat{c}_{2}
\end{array}\right], \quad S_{0}=\left[\begin{array}{ll}
0 & b \\
\bar{b} & d
\end{array}\right] .
$$

Formulas (3.1) and (3.6) yield (for $R=\left\{r_{i j}\right\}_{i, j=1}^{2}$ ) the equality

$$
A R+R A^{*}=\varkappa R+\left[\begin{array}{cc}
r_{12}+r_{21} & r_{22} \\
r_{22} & 0
\end{array}\right], \quad \varkappa:=\mu_{0}+\bar{\mu}_{0} .
$$

From the definition of $A$ we also obtain

$$
e_{A}(x, t)=\mathrm{e}^{\mu_{0} x-\mathrm{i} \mu_{0}^{2} t}\left(I_{2}+\left[\begin{array}{cc}
0 & x-2 \mathrm{i} \mu_{0} t \\
0 & 0
\end{array}\right]\right) .
$$

Assume (in addition to (3.6)) that

$$
\varkappa:=\mu_{0}+\bar{\mu}_{0}=0, \quad \widehat{c}_{1}=1, \quad \widehat{c}_{2}=0, \quad C=I_{2} .
$$

Taking into account (3.7) and the first three equalities in (3.9), we see that the relations $R=R^{*}$ and (3.1) are equivalent to the equalities

$$
r_{11}=\overline{r_{11}}, \quad r_{21}=\overline{r_{12}}, \quad r_{12}+\overline{r_{12}}=1, \quad r_{22}=0 .
$$

In view of $(3.2),(3.3),(3.8)$ and (3.9), we have

$$
\Pi(x, t)=\mathrm{e}^{\mu_{0} x-\mathrm{i} \mu_{0}^{2} t}\left[\begin{array}{l}
1 \\
0
\end{array}\right], \quad S(x, t)=S_{0}+\left[\begin{array}{cc}
1 & x-2 \mathrm{i} \mu_{0} t \\
0 & 1
\end{array}\right] R\left[\begin{array}{cc}
1 & 0 \\
x-2 \mathrm{i} \mu_{0} t & 1
\end{array}\right] .
$$

Here we took into account that $\varkappa=0$ yields $\left|\mathrm{e}^{\mu_{0} x-\mathrm{i} \mu_{0}^{2} t}\right|=1$. From (3.5), (3.6), (3.10) and (3.11), after some simple calculations we derive

$$
\begin{aligned}
& \widetilde{\Pi}(x, t)^{*}=\Pi(x, t)^{*} S(x, t)^{-1}=\left(c+d\left(x-2 \mathrm{i} \mu_{0} t\right)\right)^{-1} \mathrm{e}^{\mathrm{i} \mu_{0}^{2} t-\mu_{0} x}\left[d-r_{12}-b\right], \\
& c:=d r_{11}-\left|r_{12}+b\right|^{2}, \\
& \Pi(x, t)^{*} S(x, t)^{-1} \Pi(x, t)=d\left(c+d\left(x-2 \mathrm{i} \mu_{0} t\right)\right)^{-1}, \\
& \widetilde{q}(x, t)=2 d^{2}\left(c+d\left(x-2 \mathrm{i} \mu_{0} t\right)\right)^{-2} .
\end{aligned}
$$

Clearly, this potential $\widetilde{q}$ is rational, depends on one variable $x-2 \mathrm{i} \mu_{0} t$ and has singularity at certain values of $x, t \in \mathbb{R}$. According to Proposition 3.1, each entry of $\widetilde{\Pi}^{*}$ of the form (3.12) (in our case these entries are collinear) satisfies the Schrödinger equation with the potential $\widetilde{q}$, which is given above.

In the following example, the potential $\widetilde{q}$ is rational and depends on two real-valued variables $x$ and $t$ or, equivalently, on one complex-valued variable $P:=x-\mathrm{i} \mu_{0} t$ (and its complex conjugate $\bar{P}$ ). 
Example 3.3. Put $p=n=1, N=2, S_{0}=0$,

$$
A=\left[\begin{array}{cc}
\mu_{0} & 1 \\
0 & \mu_{0}
\end{array}\right], \quad \varkappa:=\mu_{0}+\bar{\mu}_{0}>0, \quad \widehat{C}=\left[\begin{array}{l}
0 \\
1
\end{array}\right], \quad C=\left[\begin{array}{ll}
1 & 1
\end{array}\right] .
$$

Using (3.7), we immediately check that

$$
R=\varkappa^{-1}\left[\begin{array}{cc}
2 \varkappa^{-2} & -\varkappa^{-1} \\
-\varkappa^{-1} & 1
\end{array}\right] .
$$

Taking into account (3.3), (3.8), (3.13) and (3.14), we easily calculate

$$
S(x, t)=\varkappa^{-1}\left|\mathrm{e}^{\mu_{0} P(x, t)}\right|^{2}\left(2 \varkappa^{-2}-\varkappa^{-1}(P(x, t)+\bar{P}(x, t)+2)+|P(x, t)+1|^{2}\right) .
$$

We sometimes omit the variables $x, t$ in our further formulas. In view of (3.2), (3.8), (3.13) and (3.15) we derive

$$
\Pi^{*} S^{-1} \Pi=\frac{\varkappa|P+1|^{2}}{2 \varkappa^{-2}-\varkappa^{-1}(P+\bar{P}+2)+|P+1|^{2}} .
$$

The rational potential $\widetilde{q}$, which is given by (3.5), takes the form

$$
\widetilde{q}=\frac{2\left((P+1)^{2}+(\bar{P}+1)^{2}-2 \varkappa^{-1}(P+\bar{P}+2)\right)}{\left(2 \varkappa^{-2}-\varkappa^{-1}(P+\bar{P}+2)+|P+1|^{2}\right)^{2}} .
$$

Finally, the solution $\widetilde{\Pi}^{*}=\Pi^{*} S^{-1}$ of the Schrödinger equation, where the potential $\widetilde{q}$ has the form (3.16), is given by the formula:

$$
\widetilde{\Pi}^{*}=\frac{\varkappa \mathrm{e}^{-\mu_{0} P(x, t)}\left(x+2 \mathrm{i} \bar{\mu}_{0} t+1\right)}{2 \varkappa^{-2}-\varkappa^{-1}(P(x, t)+\bar{P}(x, t)+2)+|P(x, t)+1|^{2}} .
$$

It was shown in [37] that if $\sigma(\mathrm{i} A) \subset \mathbb{C}_{+}$and the pair $A, \widehat{C}$ is full range, i.e.,

$$
\operatorname{span} \bigcup_{\ell=0}^{N-1} \operatorname{Im}\left(A^{\ell} \widehat{C}\right)=\mathbb{C}^{N}
$$

then the solution $R$ of (3.1) is unique and positive-definite, that is, $R>0$. Hence, we obtain our next proposition.

Proposition 3.4. Assume that $\sigma(\mathrm{i} A) \subset \mathbb{C}_{+}$, the pair $A, \widehat{C}$ is full range, $\operatorname{rank} C=n$ and $S_{0} \geq 0$. Then we have $S(x, t)>0$. Therefore, $S(x, t)$ is invertible and the potential $\widetilde{q}$ is nonsingular.

In our next example we deal with a nonsingular pseudo-exponential potential depending on two variables.

Example 3.5. Let the parameter matrices $A, \widehat{C}$ and $S_{0}$ have the form (3.6). Instead of the relations (3.9), we assume now that

$$
\varkappa:=\mu_{0}+\bar{\mu}_{0}>0, \quad \widehat{c}_{1}=0, \quad \widehat{c}_{2}=1, \quad b=0, \quad d>0, \quad C=I_{2} .
$$

Like in Example 3.3, formula (3.7) again yields (3.14). Taking into account (3.2), (3.3), (3.6), (3.8), (3.14) and (3.17) we calculate

$$
\begin{aligned}
& \Pi^{*} S^{-1} \Pi=Z_{1} / Z_{2}, \quad Z_{1}=2 \varkappa^{-3}+d\left|\mathrm{e}^{\mu_{0} P}\right|^{-2}|P|^{2}, \\
& Z_{2}=\varkappa^{-4}+\varkappa^{-1} d\left|\mathrm{e}^{\mu_{0} P}\right|^{-2}\left(|P|^{2}-\varkappa^{-1}(P+\bar{P})+2 \varkappa^{-2}\right), \quad P:=x-\mathrm{i} \mu_{0} t .
\end{aligned}
$$


Next, one easily obtains the derivatives of $Z_{1}$ and $Z_{2}$ with respect to $x$ :

$$
\begin{aligned}
& \left(Z_{1}\right)_{x}=-\varkappa\left(Z_{1}-2 \varkappa^{-3}\right)+d\left|\mathrm{e}^{\mu_{0} P}\right|^{-2}(P+\bar{P}), \\
& \left(Z_{2}\right)_{x}=-\varkappa\left(Z_{2}-\varkappa^{-4}\right)+\varkappa^{-1} d\left|\mathrm{e}^{\mu_{0} P}\right|^{-2}\left(P+\bar{P}-2 \varkappa^{-1}\right) .
\end{aligned}
$$

Hence, in view of (3.5) and formulas for $Z_{k}$ and $\left(Z_{k}\right)_{x}$ above, we have

$$
\begin{aligned}
\widetilde{q}= & -2\left(\Pi^{*} S^{-1} \Pi\right)_{x} \\
= & 2 \varkappa^{-2} Z_{2}-\varkappa^{-3} Z_{1}+2 \varkappa^{-2} d\left|e\left(\mu_{0}\right)\right|^{-2} Z_{1}+d\left|e\left(\mu_{0}\right)\right|^{-2}(P+\bar{P})\left(Z_{2}-\varkappa^{-1} Z_{1}\right) \\
= & -\frac{2 d\left|\mathrm{e}^{\mu_{0} P}\right|^{-2}}{Z_{2}^{2}}\left(8 \varkappa^{-5}-3 \varkappa^{-4}(P+\bar{P})\right. \\
& \left.+\varkappa^{-3}\left(|P|^{2}+2 d\left|\mathrm{e}^{\mu_{0} P}\right|^{-2}(P+\bar{P})\right)+\varkappa^{-2} d\left|\mathrm{e}^{\mu_{0} P}\right|^{-2}\left(2|P|^{2}-(P+\bar{P})^{2}\right)\right) .
\end{aligned}
$$

The solution $\widetilde{\Pi}^{*}=\Pi^{*} S^{-1}$ of (3.4) is given (in our case) by the formula

$$
\widetilde{\Pi}^{*}=\left(\mathrm{e}^{-\mu_{0} P} / Z_{2}\right)\left[\varkappa^{-2}+d\left|\mathrm{e}^{\mu_{0} P}\right|^{-2} \bar{P} \quad 2 \varkappa^{-3}-\varkappa^{-2} P\right] .
$$

\section{Nonlinear integrable equations}

Among $(2+1)$-dimensional integrable equations, Kadomtsev-Petviashvili, Davey-Stewartson (DS) and generalized nonlinear optics (also called $N$-wave) equations are, perhaps, the most actively studied systems. $S$-nodes were applied to the construction and study of the pseudoexponential, rational and nonsingular rational (so called multi-lump) solutions of the KadomtsevPetviashvili equations in [37]. Here we investigate the remaining two equations from the three above.

\subsection{Davey-Stewartson equations}

The Davey-Stewartson equations are well-known in wave theory (see, e.g., [6, 11, 23, 26] and references therein). Since Davey-Stewartson equations (DS I and DS II) are natural multidimensional generalizations of the nonlinear Schrödinger equations (NLS), their matrix versions should also be of interest (similar to matrix versions of NLS, see, e.g., [4]).

1. The matrix DS I has the form

$$
\begin{aligned}
& \mathrm{i} u_{t}-\left(u_{x x}+u_{y y}\right) / 2=u q_{1}-q_{2} u, \\
& \left(q_{1}\right)_{x}-\left(q_{1}\right)_{y}=\frac{1}{2}\left(\left(u^{*} u\right)_{y}+\left(u^{*} u\right)_{x}\right), \quad\left(q_{2}\right)_{x}+\left(q_{2}\right)_{y}=\frac{1}{2}\left(\left(u u^{*}\right)_{y}-\left(u u^{*}\right)_{x}\right),
\end{aligned}
$$

where $u, q_{1}$ and $q_{2}$ are $m_{2} \times m_{1}, m_{1} \times m_{1}$ and $m_{2} \times m_{2}$ matrix functions, respectively $\left(m_{1} \geq 1\right.$, $\left.m_{2} \geq 1\right)$. We note that another matrix version of the Davey-Stewartson equation, where $m_{1}=m_{2}$, was dealt with in [26]. It is easy to see that in the scalar case $m_{1}=m_{2}=1$ equations (4.1) and (4.2) are equivalent, for instance, to [23, p. 70, system (2.23)] (after setting in $(2.23) \varepsilon=\alpha=1)$.

GBDT version of the Bäcklund-Darboux transformation for the matrix DS I was constructed in [36]. When the initial DS I equation (in GBDT for DS I, see [36, Theorem 5]) is trivial, that is, when we set (in [36]) $u_{0} \equiv 0$ and $Q_{0} \equiv 0$, Theorem 5 from [36] takes the form:

Proposition 4.1. Let an $n \times m\left(n \in \mathbb{N}, m=m_{1}+m_{2}\right)$ matrix function $\Pi$ and an $n \times n$ matrix function $S$ satisfy equations

$$
\Pi_{x}=\Pi_{y} j, \quad \Pi_{t}=-\mathrm{i} \Pi_{y y} j, \quad j:=\left[\begin{array}{cc}
I_{m_{1}} & 0 \\
0 & -I_{m_{2}}
\end{array}\right],
$$




$$
S_{y}=-\Pi \Pi^{*}, \quad S_{x}=-\Pi j \Pi^{*}, \quad S_{t}=\mathrm{i}\left(\Pi_{y} j \Pi^{*}-\Pi j \Pi_{y}^{*}\right) .
$$

Partition $\Pi$ into $n \times m_{1}$ and $n \times m_{2}$, respectively, blocks $\Phi_{1}$ and $\Phi_{2}$ (i.e., set $\Pi=:\left[\begin{array}{ll}\Phi_{1} & \Phi_{2}\end{array}\right]$ ). Then, the matrix functions

$$
u=2 \Phi_{2}^{*} S^{-1} \Phi_{1}, \quad q_{1}=\frac{1}{2} u^{*} u-2\left(\Phi_{1}^{*} S^{-1} \Phi_{1}\right)_{y}, \quad q_{2}=-\frac{1}{2} u u^{*}+2\left(\Phi_{2}^{*} S^{-1} \Phi_{2}\right)_{y}
$$

satisfy (in the points of invertibility of S) DS I system (4.1), (4.2).

Introduce $\Phi_{1}, \Phi_{2}$ and $S$ via relations

$$
\begin{aligned}
& \Phi_{1}(x, t, y)=C_{1} E_{1}(x, t, y) \widehat{C}_{1}, \quad E_{1}(x, t, y):=\exp \left\{(x+y) A_{1}-\mathrm{i} t A_{1}^{2}\right\}, \\
& \Phi_{2}(x, t, y)=C_{2} E_{2}(x, t, y) \widehat{C}_{2}, \quad E_{2}(x, t, y):=\exp \left\{(x-y) A_{2}+\mathrm{i} t A_{2}^{2}\right\}, \\
& S(x, t, y)=S_{0}+C_{1} E_{1}(x, t, y) R_{1} E_{1}(x, t, y)^{*} C_{1}^{*} \\
& -C_{2} E_{2}(x, t, y) R_{2} E_{2}(x, t, y)^{*} C_{2}^{*}, \quad S_{0}=S_{0}^{*},
\end{aligned}
$$

where $C_{1}$ and $C_{2}$ are $n \times N$ matrices, $A_{1}, A_{2}, R_{1}=R_{1}^{*}$ and $R_{2}=R_{2}^{*}$ are $N \times N$ matrices, $\widehat{C}_{1}$ and $\widehat{C}_{2}$ are $N \times m_{1}$ and $N \times m_{2}$, respectively, matrices, $S_{0}$ is an $n \times n$ matrix and the following identities hold:

$$
A_{1} R_{1}+R_{1} A_{1}^{*}=-\widehat{C}_{1} \widehat{C}_{1}^{*}, \quad A_{2} R_{2}+R_{2} A_{2}^{*}=-\widehat{C}_{2} \widehat{C}_{2}^{*}
$$

It is immediate from (4.6)-(4.9) that $\Pi=\left[\begin{array}{ll}\Phi_{1} & \Phi_{2}\end{array}\right]$ and $S$ satisfy relations (4.3) and the first two relations in (4.4). In order to prove the third equality in (4.4), we note that

$$
\begin{aligned}
\left(C_{1} E_{1} R_{1} E_{1}^{*} C_{1}^{*}\right)_{t} & =-\mathrm{i} C_{1} E_{1}\left(A_{1}^{2} R_{1}-R_{1}\left(A_{1}^{2}\right)^{*}\right) E_{1}^{*} C_{1}^{*} \\
& =-\mathrm{i} C_{1} E_{1}\left(A_{1}\left(A_{1} R_{1}+R_{1} A_{1}^{*}\right)-\left(A_{1} R_{1}+R_{1} A_{1}^{*}\right) A_{1}^{*}\right) E_{1}^{*} C_{1}^{*} \\
& =\mathrm{i}\left(\left(\Phi_{1}\right)_{y} \Phi_{1}^{*}-\Phi_{1}\left(\Phi_{1}^{*}\right)_{y}\right) .
\end{aligned}
$$

Here we used (4.6) and the first identity in (4.9).

In a similar way we show that

$$
\left(C_{2} E_{2} R_{2} E_{2}^{*} C_{2}^{*}\right)_{t}=\mathrm{i}\left(\left(\Phi_{2}\right)_{y} \Phi_{2}^{*}-\Phi_{2}\left(\Phi_{2}^{*}\right)_{y}\right) .
$$

Equalities (4.8), (4.10) and (4.11) yield the last equality in (4.4). Hence, the conditions of Proposition 4.1 are valid, and so we proved the following proposition.

Proposition 4.2. Let $\Phi_{1}, \Phi_{2}$ and $S$ be given by the formulas (4.6)-(4.8) and assume that (4.9) holds. Then, the matrix functions $u, q_{1}$ and $q_{2}$ given by (4.5) satisfy (in the points of invertibility of $S)$ DS I system (4.1), (4.2).

Remark 4.3. It is easy to see that if $\sigma\left(A_{1}\right)=\sigma\left(A_{2}\right)=0$, then $\Phi_{1}, \Phi_{2}$ and $S$ are rational matrix functions. Thus, if $\sigma\left(A_{1}\right)=\sigma\left(A_{2}\right)=0$, the solutions $u, q_{1}$ and $q_{2}$ of the DS I system, which are constructed in Proposition 4.2, are also rational matrix functions.

Remark 4.4. Note that matrices considered in (4.9) form two separate $S$-nodes or, equivalently, an $S$-node, where $R$ is a block diagonal matrix and the matrix identity

$$
\left[\begin{array}{cc}
A_{1} & 0 \\
0 & A_{2}
\end{array}\right] R+R\left[\begin{array}{cc}
A_{1}^{*} & 0 \\
0 & A_{2}^{*}
\end{array}\right]=-\left[\begin{array}{cc}
\widehat{C}_{1} \widehat{C}_{1}^{*} & 0 \\
0 & \widehat{C}_{2} \widehat{C}_{2}^{*}
\end{array}\right], \quad R:=\left[\begin{array}{cc}
R_{1} & 0 \\
0 & R_{2}
\end{array}\right]
$$

is valid. Another example of a block diagonal matrix $R$ is dealt with in Subsection 4.2. It would also be of interest to compare solutions of the same system constructed using $r_{1}$-nodes and $r_{2}$-nodes $\left(r_{1} \neq r_{2}\right)$. 
2. The compatibility condition $w_{t x}=w_{x t}$ of the auxiliary systems

$$
w_{x}= \pm \mathrm{i} j w_{y}+j V w, \quad w_{t}=2 \mathrm{i} j w_{y y} \pm 2 j V w_{y} \pm j Q w
$$

where

$$
\begin{aligned}
& V=\left[\begin{array}{cc}
0 & u \\
u^{*} & 0
\end{array}\right], \quad Q=\left[\begin{array}{cc}
q_{1} & u_{y} \mp \mathrm{i} u_{x} \\
u_{y}^{*} \pm \mathrm{i} u_{x}^{*} & -q_{2}
\end{array}\right], \\
& q_{k}(x, t)=-q_{k}(x, t)^{*}, \quad k=1,2,
\end{aligned}
$$

is equivalent (for the case that the solution $w$ is a non-degenerate matrix function) to the matrix DS II equation

$$
\begin{aligned}
& u_{t}+\mathrm{i}\left(u_{x x}-u_{y y}\right)= \pm\left(q_{1} u-u q_{2}\right), \\
& \left(q_{1}\right)_{x} \mp \mathrm{i}\left(q_{1}\right)_{y}=\left(u u^{*}\right)_{y} \mp \mathrm{i}\left(u u^{*}\right)_{x}, \quad\left(q_{2}\right)_{x} \pm \mathrm{i}\left(q_{2}\right)_{y}=\left(u^{*} u\right)_{y} \pm \mathrm{i}\left(u^{*} u\right)_{x} .
\end{aligned}
$$

As we see from (4.12)-(4.16), there are two versions of auxiliary systems and corresponding DS II equations. After setting $m_{1}=m_{2}=1$ (and setting also $\varepsilon=1, \alpha=\mp \mathrm{i}$ in [23, p. 70, system (2.23)]), like for the scalar DS I case, equations (4.15) and (4.16) are equivalent to [23, p. $70,(2.23)]$.

Open problem. Use the approach from Proposition 4.1 in order to construct explicit pseudoexponential solutions of the matrix DS II.

We note that various results on DS II, including BDT results, are not quite analogous to the results on DS I (see, e.g., [23]). A quasi-determinant approach to explicit solution of noncommutative DS equations is presented in [19].

\subsection{Generalized nonlinear optics equation}

The integrability of the generalized nonlinear optics equation (GNOE)

$$
\begin{aligned}
& {\left[D, \xi_{t}\right]-\left[\widetilde{D}, \xi_{x}\right]=[[D, \xi],[\widetilde{D}, \xi]]+D \xi_{y} \widetilde{D}-\widetilde{D} \xi_{y} D,} \\
& \xi(x, t, y)^{*}=B \xi(x, t, y) B, \quad B=\operatorname{diag}\left\{b_{1}, b_{2}, \ldots, b_{m}\right\}, \quad b_{k}= \pm 1, \\
& D=\operatorname{diag}\left\{d_{1}, d_{2}, \ldots, d_{m}\right\}>0, \quad \widetilde{D}=\operatorname{diag}\left\{\widetilde{d}_{1}, \widetilde{d}_{2}, \ldots, \widetilde{d}_{m}\right\}>0
\end{aligned}
$$

was dealt with in $[2,53]$. This system is a generalization of the well-known $N$-wave (nonlinear optics) equation $\left[D, \xi_{t}\right]-\left[\widetilde{D}, \xi_{x}\right]=[[D, \xi],[\widetilde{D}, \xi]]$ first studied in [51] (see also [3]). GBDT version of the Bäcklund-Darboux transformation for GNOE was constructed in [36]. When the initial system in GBDT for GNOE [36, Theorem 4] is trivial (i.e., $\xi_{0} \equiv 0$ ), Theorem 4 from [36] takes the form:

Proposition 4.5. Let an $n \times m$ matrix function $\Pi$ and an $n \times n$ matrix function $S$ satisfy equations

$$
\begin{aligned}
& \Pi_{x}=\Pi_{y} D, \quad \Pi_{t}=\Pi_{y} \widetilde{D}, \\
& S_{y}=-\Pi B \Pi^{*}, \quad S_{x}=-\Pi B D \Pi^{*}, \quad S_{t}=-\Pi B \widetilde{D} \Pi^{*} .
\end{aligned}
$$

Then the matrix function

$$
\xi=\Pi^{*} S^{-1} \Pi B
$$

satisfies (in the points of invertibility of S) GNOE (4.17) and reduction condition (4.18). 
In order to construct pseudo-exponential-type solutions $\xi$, we will consider matrix functions $\Pi$ and $S$ of the form (2.1) and (2.5), respectively, where $E_{A}$ will depend on three variables and $N=m l, l \in \mathbb{N}$. Namely, we set

$$
\begin{aligned}
& \Pi(x, t, y)=C E_{A}(x, t, y) \widehat{C}, \quad E_{A}(x, t, y)=\exp \left\{x A_{1}+t A_{2}+y A_{3}\right\}, \\
& A_{1}=D \otimes A, \quad A_{2}=\widetilde{D} \otimes A, \quad A_{3}=I_{m} \otimes A, \\
& \widehat{C}=\sum_{k=1}^{m}\left(e_{k} e_{k}^{*}\right) \otimes\left(\widehat{c} e_{k}\right), \quad e_{k}=\left\{\delta_{i k}\right\}_{i=1}^{m} \in \mathbb{C}^{m},
\end{aligned}
$$

where $C$ is an $n \times N$ matrix, $A$ is an $l \times l$ matrix, $N=m l, \otimes$ is Kronecker product, $\widehat{c}$ is an $l \times m$ matrix, $e_{k}$ is a column vector and $\delta_{i k}$ is Kronecker's delta. It is immediate that the matrices $A_{k}$ $(k=1,2,3)$ commute. Hence, we see that matrices $A, C$ and $\widehat{c}$ determine (via (4.21)-(4.23)) matrix function $\Pi$ satisfying (4.19).

Proposition 4.6. Let relations (4.21)-(4.23) hold and set

$$
S(x, t, y)=S_{0}+C E_{A}(x, t, y) R E_{A}(x, t, y)^{*} C^{*}, \quad S_{0}=S_{0}^{*},
$$

where the $N \times N$ matrix $R\left(N=m l, R=R^{*}\right)$ satisfies matrix identities

$$
\begin{aligned}
& A_{1} R+R A_{1}^{*}=-\widehat{C} B D \widehat{C}^{*}, \quad A_{2} R+R A_{2}^{*}=-\widehat{C} B \widetilde{D} \widehat{C}^{*}, \\
& A_{3} R+R A_{3}^{*}=-\widehat{C} B \widehat{C}^{*} .
\end{aligned}
$$

Then, the matrix function $\xi=\Pi^{*} S^{-1} \Pi B$ satisfies (in the points of invertibility of $S$ ) GNOE (4.17) and reduction condition (4.18).

Proof. We mentioned above that $\Pi$ given by (4.21)-(4.23) satisfies (4.19). Moreover, relations (4.21) and (4.24)-(4.26) yield (4.20). Thus, the conditions of Proposition 4.5 are fulfilled.

We note that, according to (4.23), the right-hand sides of the equalities in (4.25) and (4.26) are block diagonal matrices with $l \times l$ blocks. Therefore, we will construct block diagonal matrix $R$, the blocks $R_{k k}$ of which are also $l \times l$ matrices:

$$
R=\operatorname{diag}\left\{R_{11}, R_{22}, \ldots, R_{m m}\right\} .
$$

Taking into account (4.22), we see that for $R$ of the form (4.27) identities

$$
A R_{k k}+R_{k k} A^{*}=-b_{k}\left(\widehat{c} e_{k}\right)\left(\widehat{c} e_{k}\right)^{*}, \quad 1 \leq k \leq m,
$$

imply that identities (4.25) and (4.26) hold.

Corollary 4.7. Let relations (4.21)-(4.23) and (4.28) hold. Then, the matrix function $\xi=$ $\Pi^{*} S^{-1} \Pi B$, where $S$ is given by (4.24) and (4.27), satisfies (in the points of invertibility of $S$ ) GNOE (4.17) and reduction condition (4.18).

Remark 4.8. If $\sigma(A) \cap \sigma\left(-A^{*}\right)=\varnothing$, there exist unique solutions $R_{k k}$ satisfying (4.28). For that case we have also $R_{k k}=R_{k k}^{*}$ (i.e., $R=R^{*}$ ). Clearly, $R_{k k}$ is immediately recovered if $\sigma(A) \cap \sigma\left(-A^{*}\right)=\varnothing$ and $A$ is a diagonal matrix.

\section{Acknowledgements}

The research of I.Ya. Roitberg was supported by the German Research Foundation (DFG) under grant No. KI 760/3-1. The research of A.L. Sakhnovich was supported by the Austrian Science Fund (FWF) under Grant No. P24301. 


\section{References}

[1] Ablowitz M.J., Chakravarty S., Trubatch A.D., Villarroel J., A novel class of solutions of the non-stationary Schrödinger and the Kadomtsev-Petviashvili I equations, Phys. Lett. A 267 (2000), 132-146.

[2] Ablowitz M.J., Haberman R., Nonlinear evolution equations - two and three dimensions, Phys. Rev. Lett. 35 (1975), 1185-1188.

[3] Ablowitz M.J., Haberman R., Resonantly coupled nonlinear evolution equations, J. Math. Phys. 16 (1975), 2301-2305.

[4] Ablowitz M.J., Prinari B., Trubatch A.D., Discrete and continuous nonlinear Schrödinger systems, London Mathematical Society Lecture Note Series, Vol. 302, Cambridge University Press, Cambridge, 2004.

[5] Andrianov A.A., Borisov N.V., Ioffe M.V., The factorization method and quantum systems with equivalent energy spectra, Phys. Lett. A 105 (1984), 19-22.

[6] Anker D., Freeman N.C., On the soliton solutions of the Davey-Stewartson equation for long waves, Proc. Roy. Soc. London Ser. A 360 (1978), 529-540.

[7] Bagrov V.G., Samsonov B.F., Supersymmetry of a nonstationary Schrödinger equation, Phys. Lett. A 210 (1996), 60-64.

[8] Bergvelt M., Gekhtman M., Kasman A., Spin Calogero particles and bispectral solutions of the matrix KP hierarchy, Math. Phys. Anal. Geom. 12 (2009), 181-200, arXiv:0806.2613.

[9] Caudrey P.J., Gibbon J.D., Eilbeck J.C., Bullough R.K., Exact multisoliton solutions of the self-induced transparency and sine-Gordon equations, Phys. Rev. Lett. 30 (1973), 237-238.

[10] Cieśliński J.L., Algebraic construction of the Darboux matrix revisited, J. Phys. A: Math. Theor. 42 (2009), 404003, 40 pages, arXiv:0904.3987.

[11] Davey A., Stewartson K., On three-dimensional packets of surface waves, Proc. Roy. Soc. London Ser. A 338 (1974), 101-110.

[12] Dimakis A., Müller-Hoissen F., Solutions of matrix NLS systems and their discretizations: a unified treatment, Inverse Problems 26 (2010), 095007, 55 pages, arXiv:1001.0133.

[13] Dimakis A., Müller-Hoissen F., Binary Darboux transformations in bidifferential calculus and integrable reductions of vacuum Einstein equations, SIGMA 9 (2013), 009, 31 pages, arXiv:1207.1308.

[14] Fritzsche B., Kirstein B., Roitberg I.Ya., Sakhnovich A.L., Weyl theory and explicit solutions of direct and inverse problems for Dirac system with a rectangular matrix potential, Oper. Matrices 7 (2013), 183-196, arXiv:1105.2013.

[15] Fritzsche B., Kirstein B., Sakhnovich A.L., Completion problems and scattering problems for Dirac type differential equations with singularities, J. Math. Anal. Appl. 317 (2006), 510-525, math.SP/0409424.

[16] Gesztesy F., Holden H., Soliton equations and their algebro-geometric solutions. Vol. I. (1+1)-dimensional continuous models, Cambridge Studies in Advanced Mathematics, Vol. 79, Cambridge University Press, Cambridge, 2003.

[17] Gesztesy F., Holden H., Michor J., Teschl G., Soliton equations and their algebro-geometric solutions. Vol. II. $(1+1)$-dimensional discrete models, Cambridge Studies in Advanced Mathematics, Vol. 114, Cambridge University Press, Cambridge, 2008.

[18] Gesztesy F., Teschl G., On the double commutation method, Proc. Amer. Math. Soc. 124 (1996), 1831-1840.

[19] Gilson C.R., Macfarlane S.R., Dromion solutions of noncommutative Davey-Stewartson equations, J. Phys. A: Math. Theor. 42 (2009), 235202, 20 pages, arXiv:0901.4918.

[20] Gohberg I., Kaashoek M.A., Sakhnovich A.L., Canonical systems with rational spectral densities: explicit formulas and applications, Math. Nachr. 194 (1998), 93-125.

[21] Gohberg I., Kaashoek M.A., Sakhnovich A.L., Pseudo-canonical systems with rational Weyl functions: explicit formulas and applications, J. Differential Equations 146 (1998), 375-398.

[22] Gohberg I., Kaashoek M.A., Sakhnovich A.L., Scattering problems for a canonical system with a pseudoexponential potential, Asymptot. Anal. 29 (2002), 1-38.

[23] Gu C., Hu H., Zhou Z., Darboux transformations in integrable systems. Theory and their applications to geometry, Mathematical Physics Studies, Vol. 26, Springer, Dordrecht, 2005.

[24] Hirota R., Exact solution of the sine-Gordon equation for multiple collisions of solitons, J. Phys. Soc. Japan 33 (1972), 1459-1463.

[25] Kasman A., Gekhtman M., Solitons and almost-intertwining matrices, J. Math. Phys. 42 (2001), 3540-3551, math-ph/0011011. 
[26] Leznov A.N., Yuzbashyan E.A., Multi-soliton solutions of the two-dimensional matrix Davey-Stewartson equation, Nuclear Phys. B 496 (1997), 643-653, hep-th/9612107.

[27] Livšic M.S., Operator waves in Hilbert space and related partial differential equations, Integral Equations Operator Theory 2 (1979), 25-47.

[28] Loewner C., A transformation theory of the partial differential equations of gas dynamics, Tech. Notes Nat. Adv. Comm. Aeronaut. 1950 (1950), no. 2065, 1-56.

[29] Loewner C., Generation of solutions of systems of partial differential equations by composition of infinitesimal Baecklund transformations, J. Analyse Math. 2 (1953), 219-242.

[30] Marchenko V.A., Nonlinear equations and operator algebras, Mathematics and its Applications (Soviet Series), Vol. 17, D. Reidel Publishing Co., Dordrecht, 1988.

[31] Matveev V.B., Darboux transformation and explicit solutions of the Kadomtcev-Petviaschvily equation, depending on functional parameters, Lett. Math. Phys. 3 (1979), 213-216.

[32] Matveev V.B., Salle M.A., Darboux transformations and solitons, Springer Series in Nonlinear Dynamics, Springer-Verlag, Berlin, 1991.

[33] Pecheritsyn A.A., Pozdeeva E.O., Samsonov B.F., The Darboux transformation for the nonstationary Dirac equation, Russ. Phys. J. 48 (2005), 365-374.

[34] Sabatier P.C., On multidimensional Darboux transformations, Inverse Problems 14 (1998), 355-366.

[35] Sakhnovich A.L., Exact solutions of nonlinear equations and the method of operator identities, Linear Algebra Appl. 182 (1993), 109-126.

[36] Sakhnovich A.L., Dressing procedure for solutions of nonlinear equations and the method of operator identities, Inverse Problems 10 (1994), 699-710.

[37] Sakhnovich A.L., Matrix Kadomtsev-Petviashvili equation: matrix identities and explicit non-singular solutions, J. Phys. A: Math. Gen. 36 (2003), 5023-5033.

[38] Sakhnovich A.L., Bäcklund-Darboux transformation for non-isospectral canonical system and RiemannHilbert problem, SIGMA 3 (2007), 054, 11 pages, math-ph/0703072.

[39] Sakhnovich A.L., On the GBDT version of the Bäcklund-Darboux transformation and its applications to linear and nonlinear equations and Weyl theory, Math. Model. Nat. Phenom. 5 (2010), 340-389, arXiv:0909.1537.

[40] Sakhnovich A.L., The time-dependent Schrödinger equation of dimension $k+1$ : explicit and rational solutions via GBDT and multinodes, J. Phys. A: Math. Theor. 44 (2011), 475201, 12 pages, arXiv:1104.2554.

[41] Sakhnovich A.L., Sakhnovich L.A., Roitberg I.Ya., Inverse problems and nonlinear evolution equations. Solutions, Darboux matrices and Weyl-Titchmarsh functions, De Gruyter Studies in Mathematics, Vol. 47, De Gruyter, Berlin, 2013.

[42] Sakhnovich L.A., On the factorization of the transfer matrix function, Sov. Math. Dokl. 17 (1976), $203-207$.

[43] Sakhnovich L.A., Integral equations with difference kernels on finite intervals, Operator Theory: Advances and Applications, Vol. 84, Birkhäuser Verlag, Basel, 1996.

[44] Sakhnovich L.A., Spectral theory of canonical differential systems. Method of operator identities, Operator Theory: Advances and Applications, Vol. 107, Birkhäuser Verlag, Basel, 1999.

[45] Sakhnovich L.A., Levy processes, integral equations, statistical physics: connections and interactions, Operator Theory: Advances and Applications, Vol. 225, Birkhäuser/Springer Basel AG, Basel, 2012.

[46] Schiebold C., Explicit solution formulas for the matrix-KP, Glasg. Math. J. 51 (2009), 147-155.

[47] Schief W.K., Rogers C., Loewner transformations: adjoint and binary Darboux connections, Stud. Appl. Math. 100 (1998), 391-422.

[48] Skljanin E.K., A class of potentials for the nonstationary Dirac equation, Zap. Nauchn. Sem. Leningrad. Otdel. Mat. Inst. Steklov. (LOMI) 77 (1978), 214-226.

[49] Suzko A.A., Schulze-Halberg A., Darboux transformations and supersymmetry for the generalized Schrödinger equations in $(1+1)$ dimensions, J. Phys. A: Math. Theor. 42 (2009), 295203, 14 pages.

[50] Takebe T., Dispersionless BKP hierarchy and quadrant Löwner equation, SIGMA 10 (2014), 023, 13 pages, arXiv:1308.4584.

[51] Zakharov V.E., Manakov S.V., The theory of resonance interaction of wave packets in nonlinear media, Soviet Phys. JETP 69 (1975), 1654-1673.

[52] Zakharov V.E., Mikhailov A.V., On the integrability of classical spinor models in two-dimensional spacetime, Comm. Math. Phys. 74 (1980), 21-40.

[53] Zakharov V.E., Shabat A.B., A scheme for integrating the nonlinear equations of mathematical physics by the method of the inverse scattering problem. I, Funct. Anal. Appl. 8 (1974), 226-235. 